\title{
Characterizations of Framed Curves in Four-Dimensional Euclidean Space
}

\author{
Bahar Doğan Yazıcı ${ }^{*}$, Sıddıka Özkaldı Karakuş ${ }^{1}$ and Murat Tosun ${ }^{2}$ \\ ${ }^{1}$ Department of Mathematics, Faculty of Arts and Sciences, Bilecik Şeyh Edebali University, Bilecik, Turkey \\ ${ }^{2}$ Department of Mathematics, Faculty of Arts and Sciences, Sakarya University, Sakarya, Turkey \\ * Corresponding author
}

Article Info

Keywords: Bishop-type framed curves, Euler angles, Framed curves in $\mathbb{R}^{4}$ 2010 AMS: 53A04, 58K05

Received: 11 October 2021

Accepted: 29 December 2021

Available online: 30 December 2021

\begin{abstract}
Framed curves in Euclidean space are used to investigate singular curves and are important for singularity theory. In this study, we investigate framed curves in four-dimensional Euclidean space. We introduce Bishop-type frame of framed curves is with the help of Euler angles. Also, we give framed rectifying curves, framed osculating curves and framed normal curves with the help of Bishop-type framed curves in four-dimensional Euclidean space. Also, we obtain some characterizations depending on framed curvatures.
\end{abstract}

\section{Introduction}

Framed curves in $n$-dimensional Euclidean space were first introduced by Honda and Takahashi [1]. Framed curves in Euclidean space are used to investigate singular curves and are important for singularity theory. A framed curve in the Euclidean space is a curve with a moving frame. It is a generalization not only of regular curves with linear independent condition but also of Legendre curves in the unit tangent bundle. There are many studies in the literature for framed curves in three-dimensional Euclidean space. In three-dimensional Euclidean space, there are studies such as existence and uniqueness conditions of framed curves [2], evolutes of framed immersions [3], framed rectifying curves [4,5], framed normal curves [6], Bertrand and Mannheim curves of framed curves [7].

Frenet and Bishop frames are important in classical differential geometry. Frenet frames cannot be built as the curvatures vanishes at some points and the Bishop frame is used [8]. In [9], this situation is extended to the four-dimensional Euclidean space and a parallel frame is formed. In four-dimensional Euclidean space, this frame is called the parallel transport frame and it can be [10]- [13] etc. studies are available.

In this paper, we introduce framed curves in four-dimensional Euclidean space. Also, we give some new results for the relation of framed curves with Frenet curves in four-dimensional Euclidean space. Moreover, we introduce Bishop-type frame of framed curves with the help of Euler angles. Also, by using Bishop-type framed curves in four-dimensional Euclidean space, we introduce framed rectifying curves, framed osculating curves and framed normal curves.

\section{Framed curves in $n$-Euclidean space}

A framed curve in the $n$-dimensional Euclidean space is a space curve with a moving frame which may have singular points, in detail see [1]. Let $\gamma: I \rightarrow \mathbb{R}^{n}$ be a curve with singular points. The set

$$
\Delta_{n-1}=\left\{\mu=\left(\mu_{1}, \mu_{2}, \ldots, \mu_{n-1}\right) \in \mathbb{R}^{n} \times \ldots \times \mathbb{R}^{n} \mid\left\langle\mu_{i}, \mu_{j}\right\rangle=\delta_{i j}, \quad i, j=1,2 \ldots, n-1\right\}
$$

is an $(n, n-1)$-type Stiefel manifold. Let $\mu=\left(\mu_{1}, \mu_{2}, \ldots, \mu_{n-1}\right) \in \Delta_{n-1}$. Take the unit vector defined by $v=\mu_{1} \wedge \ldots \wedge \mu_{n-1}$.

Definition 2.1 (Framed curve). $(\gamma, \mu): I \rightarrow \mathbb{R}^{n} \times \Delta_{n-1}$ is called a framed curve if $\left\langle\gamma^{\prime}(s), \mu_{i}(s)\right\rangle=0$ for all $s \in I$ and $i=1,2, \ldots, n-1[1]$. 
Let $(\gamma, \mu): I \rightarrow \mathbb{R}^{n} \times \Delta_{n-1}$ be a framed curve and $\nu(s)=\mu_{1}(s) \wedge \ldots \wedge \mu_{n-1}$. By definition $(\mu(s), v(s)) \in S O(n)$ for each $s \in I$ and $\{\mu(s), v(s)\}$ is called a moving frame along the framed base curve $\gamma(s)$. Then, Frenet-Serret type formula is given by

$$
\left(\begin{array}{l}
\mu^{\prime}(s) \\
v^{\prime}(s)
\end{array}\right)=A(s)\left(\begin{array}{c}
\mu(s) \\
v(s)
\end{array}\right)
$$

where $A(s)=\left(a_{i, j}\right) \in o(n)$ for $i, j=1,2, \ldots, n$ and $o(n)$ is the set of all skew-symmetric matrices. Moreover, there exists a smooth mapping $\alpha: I \rightarrow \mathbb{R}$ such that:

$$
\gamma^{\prime}(s)=\alpha(s) v(s)
$$

In addition, $s_{0}$ is a singular point of the framed curve $\gamma$ if and only if $\alpha\left(s_{0}\right)=0 .(A, \alpha): I \rightarrow o(n) \times \mathbb{R}$ is called the curvature of the framed curve.

\section{Framed curves in $\mathbb{R}^{4} \times \Delta_{3}$}

Let us take the vectors $x=\left(x_{1}, x_{2}, x_{3}, x_{4}\right), y=\left(y_{1}, y_{2}, y_{3}, y_{4}\right)$ and $z=\left(z_{1}, z_{2}, z_{3}, z_{4}\right)$ in four-dimensional Euclidean space $\mathbb{R}^{4}$ with Euclidean inner product. The ternary product (or vector product) is defined

$$
x \wedge y \wedge z=\left|\begin{array}{llll}
e_{1} & e_{2} & e_{3} & e_{4} \\
x_{1} & x_{2} & x_{3} & x_{4} \\
y_{1} & y_{2} & y_{3} & y_{4} \\
z_{1} & z_{2} & z_{3} & z_{4}
\end{array}\right|
$$

where $\left\{e_{1}, e_{2}, e_{3}, e_{4}\right\}$ is the standard basis of $\mathbb{R}^{4}$ [14].

The set

$$
\Delta_{3}=\left\{\mu=\left(\mu_{1}, \mu_{2}, \mu_{3}\right) \in \mathbb{R}^{4} \times \mathbb{R}^{4} \times \mathbb{R}^{4} \mid \quad\left\langle\mu_{i}, \mu_{j}\right\rangle=\delta_{i j}, i, j=1,2,3\right\}
$$

is a six-dimensional smooth manifold. We define a unit vector $v: \mu_{1} \wedge \mu_{2} \wedge \mu_{3}$ of $\mathbb{R}^{4}$.

Definition 3.1. $\left(\gamma, \mu_{1}, \mu_{2}, \mu_{3}\right): I \rightarrow \mathbb{R}^{4} \times \Delta_{3}$ is called a framed curve if $\left\langle\gamma^{\prime}(s), \mu_{i}(s)\right\rangle=0$ for all $s \in I$ and $i=1,2,3$.

The Frenet-Serret type formula is given by

$$
\left(\begin{array}{c}
v^{\prime}(s) \\
\mu_{1}^{\prime}(s) \\
\mu_{2}^{\prime}(s) \\
\mu_{3}^{\prime}(s)
\end{array}\right)=\left(\begin{array}{cccc}
0 & -l(s) & -m(s) & -n(s) \\
l(s) & 0 & p(s) & q(s) \\
m(s) & -p(s) & 0 & r(s) \\
n(s) & -q(s) & -r(s) & 0
\end{array}\right)\left(\begin{array}{l}
v(s) \\
\mu_{1}(s) \\
\mu_{2}(s) \\
\mu_{3}(s)
\end{array}\right)
$$

where

$$
\begin{array}{ll}
l(s)=\left\langle\mu_{1}^{\prime}(s), v(s)\right\rangle & m(s)=\left\langle\mu_{2}^{\prime}(s), v(s)\right\rangle \\
n(s)=\left\langle\mu_{3}^{\prime}(s), v(s)\right\rangle & p(s)=\left\langle\mu_{1}^{\prime}(s), \mu_{2}(s)\right\rangle \\
q(s)=\left\langle\mu_{1}^{\prime}(s), \mu_{3}(s)\right\rangle & r(s)=\left\langle\mu_{2}^{\prime}(s), \mu_{3}(s)\right\rangle
\end{array}
$$

In addition, there exists a smooth mapping $\alpha: I \rightarrow \mathbb{R}$ such that $\gamma^{\prime}(s)=\alpha(s) v(s)$. If $s_{0}$ is a singular point of $\gamma, \alpha\left(s_{0}\right)=0$. If $s_{0}$ is a singular point of $(\gamma, \mu),(l, m, n, p, q, r, \alpha)\left(s_{0}\right)=0$. Note that $\left(\gamma, \mu_{1}, \mu_{2}, \mu_{3}\right)$ is a framed immersion if and only if $(l(s), m(s), n(s), p(s), q(s), r(s), \alpha(s)) \neq$ $(0,0,0,0)$ for all $s \in I$.

Example 3.2. Regular curves at $\mathbb{R}^{4}$ with linear independent conditions are a natural example of framed curves $\left(\right.$ i.e. $\gamma^{\prime}(s), \gamma^{\prime \prime}(s), \gamma^{\prime \prime \prime}(s)$ are linear independent for all $s \in I) . \gamma: I \rightarrow \mathbb{R}^{4}$ regular curve with linear independent conditions. If we take $\mu_{1}(s)=N_{1}(s), \mu_{2}(s)=N_{2}(s)$ and $\mu_{3}(s)=N_{3}(s)$, then $\left(\gamma, N_{1}, N_{2}, N_{3}\right): I \rightarrow \mathbb{R}^{4} \times \Delta_{3}$ is a framed curve. Also, $v(s)=\mu_{1} \wedge \mu_{2} \wedge \mu_{3}=T(s)$. Therefore,

$$
\begin{aligned}
T(s) & =\frac{\gamma^{\prime}(s)}{\left\|\gamma^{\prime}(s)\right\|} & N_{1}(s) & =\frac{\left\|\gamma^{\prime}(s)\right\|^{2} \gamma^{\prime \prime}(s)-\left\langle\gamma^{\prime}(s), \gamma^{\prime \prime}(s)\right\rangle \gamma^{\prime}(s)}{\|\| \gamma^{\prime}(s)\left\|^{2} \gamma^{\prime \prime}(s)-\left\langle\gamma^{\prime}(s), \gamma^{\prime \prime}(s)\right\rangle \gamma^{\prime}(s)\right\|} \\
N_{2}(s) & =N_{3}(s) \times T(s) \times N_{1}(s) & N_{3}(s) & =\frac{T(s) \times N_{1}(s) \times \gamma^{\prime \prime}(s)}{\left\|T(s) \times N_{1}(s) \times \gamma^{\prime \prime \prime}(s)\right\|}
\end{aligned}
$$

\subsection{Framed curves $\mathbb{R}^{4} \times \Delta_{3}$ with Bishop frame}

In this section, adapted frame for framed curves are obtained by using Euler angles and these frame is called Bishop-type frame of framed curves. By using Euler angles an arbitrary rotation matrix is given by

$$
\left(\begin{array}{ccc}
\cos \theta \cos \psi & -\cos \varphi \sin \psi+\sin \varphi \cos \psi \sin \theta & \sin \varphi \sin \psi+\cos \varphi \cos \psi \sin \theta \\
\cos \theta \sin \psi & \cos \varphi \cos \psi+\sin \varphi \sin \psi \sin \theta & -\sin \varphi \cos \psi+\cos \varphi \sin \psi \sin \theta \\
-\sin \theta & \sin \varphi \cos \theta & \cos \varphi \cos \theta
\end{array}\right)
$$


where $\theta, \psi, \varphi$ are Euler angles [9]. We define $\left(\bar{\mu}_{1}(s), \bar{\mu}_{2}(s), \bar{\mu}_{3}(s)\right) \in \Delta_{3}$ by

$$
\begin{aligned}
\bar{\mu}_{1}(s) & =(\cos \theta(s) \cos \psi(s)) \mu_{1}(s)+(-\cos \varphi(s) \sin \psi(s)+\sin \varphi(s) \cos \psi(s) \sin \theta(s)) \mu_{2}(s) \\
& +(\sin \varphi(s) \sin \psi(s)+\cos \varphi(s) \cos \psi(s) \sin \theta(s)) \mu_{3}(s) \\
\bar{\mu}_{2}(s) & =(\cos \theta(s) \sin \psi(s)) \mu_{1}(s)+(\cos \varphi(s) \cos \psi(s)+\sin \varphi(s) \sin \psi(s) \sin \theta(s)) \mu_{2}(s) \\
& +(-\sin \varphi(s) \cos \psi(s)+\cos \varphi(s) \sin \psi(s) \sin \theta(s)) \mu_{3}(s) \\
& \\
\bar{\mu}_{3}(s) & =-\sin \theta(s) \mu_{1}(s)+\sin \varphi(s) \cos \theta(s) \mu_{2}(s)+\cos \varphi(s) \cos \theta(s) \mu_{3}(s)
\end{aligned}
$$

Therefore, $\left(\gamma, \bar{\mu}_{1}, \bar{\mu}_{2}, \bar{\mu}_{3}\right): I \rightarrow \mathbb{R}^{4} \times \Delta_{3}$ is a framed curve and

$$
\bar{v}(s)=\bar{\mu}_{1}(s) \wedge \bar{\mu}_{2}(s) \wedge \bar{\mu}_{3}(s)=v(s)
$$

By differentiating equations 3.1 and 3.2, we get

$$
\begin{aligned}
\bar{\mu}_{1}^{\prime}= & (l \cos \theta \cos \psi-m \cos \varphi \sin \psi+m \sin \varphi \cos \psi \sin \theta+n \sin \varphi \sin \psi+n \cos \varphi \cos \psi \sin \theta) v \\
& +\left(-\theta^{\prime} \sin \theta \cos \psi-\psi^{\prime} \cos \theta \sin \psi+p \cos \varphi \sin \psi-p \sin \varphi \cos \psi \sin \theta\right. \\
& -q \sin \varphi \sin \psi-q \cos \varphi \sin \theta \cos \psi) \mu_{1}+\left(p \cos \theta \cos \psi+\varphi^{\prime} \sin \varphi \sin \psi-\psi^{\prime} \cos \varphi \cos \psi\right. \\
& +\varphi^{\prime} \sin \theta \cos \varphi \cos \psi+\theta^{\prime} \cos \theta \sin \varphi \cos \psi-\psi^{\prime} \sin \theta \sin \varphi \sin \psi-r \sin \varphi \sin \psi \\
& -r \cos \varphi \cos \psi \sin \theta) \mu_{2}+(q \cos \theta \cos \psi-r \cos \varphi \sin \psi+r \sin \varphi \cos \psi \sin \theta \\
& +\varphi^{\prime} \cos \varphi \sin \psi+\psi^{\prime} \sin \varphi \cos \psi-\varphi^{\prime} \sin \theta \sin \varphi \cos \psi+\theta^{\prime} \cos \theta \cos \varphi \cos \psi \\
& \left.-\psi^{\prime} \sin \theta \cos \varphi \sin \psi\right) \mu_{3}, \\
& \\
\bar{\mu}_{2}^{\prime}=\quad & l \cos \theta \sin \psi+m \cos \varphi \cos \psi+m \sin \varphi \sin \psi \sin \theta-n \sin \varphi \cos \psi+n \cos \varphi \sin \psi \sin \theta) v \\
+ & \left(-\theta^{\prime} \sin \theta \sin \psi+\psi^{\prime} \cos \theta \cos \psi-p \cos \varphi \cos \psi-p \sin \varphi \sin \psi \sin \theta\right. \\
+ & q \sin \varphi \cos \psi-q \cos \varphi \sin \theta \sin \psi) \mu_{1}+\left(p \cos \theta \sin \psi-\psi^{\prime} p \cos \varphi \sin \psi+\psi^{\prime} \sin \varphi \cos \psi \sin \theta\right. \\
- & \varphi^{\prime} \cos \psi \sin \varphi+\varphi^{\prime} \sin \theta \cos \varphi \sin \psi+\theta^{\prime} \cos \theta \sin \varphi \sin \psi+r \sin \varphi \cos \psi \\
- & r \cos \varphi \sin \psi \sin \theta) \mu_{2}+(q \cos \theta \sin \psi+r \cos \varphi \cos \psi+r \sin \varphi \sin \psi \sin \theta \\
- & \varphi^{\prime} \cos \varphi \cos \psi+\psi^{\prime} \sin \varphi \sin \psi-\varphi^{\prime} \sin \theta \sin \varphi \sin \psi+\theta^{\prime} \cos \theta \cos \varphi \sin \psi \\
+ & \left.\psi^{\prime} \sin \theta \cos \varphi \cos \psi\right) \mu_{3}, \\
& \\
\bar{\mu}_{3}^{\prime}= & (-l \sin \theta+m \sin \varphi \cos \theta+n \cos \varphi \cos \theta) v+\left(-\theta^{\prime} \cos \theta-p \sin \varphi \cos \theta-q \cos \varphi \cos \theta\right) \mu_{1} \\
& +\left(-p \sin \theta+\varphi^{\prime} \cos \theta \cos \varphi-\theta^{\prime} \sin \theta \sin \varphi-r \cos \varphi \cos \theta\right) \mu_{2} \\
& +\left(-q \sin \theta+r \sin \varphi \cos \theta-\varphi^{\prime} \sin \varphi \cos \theta+\theta^{\prime} \sin \theta \cos \varphi\right) \mu_{3}, \\
\bar{v}^{\prime} \quad= & v^{\prime}=-l \mu_{1}-m \mu_{2}-n \mu_{3} .
\end{aligned}
$$

Corollary 3.3. If we take Euler angles $\theta, \psi, \varphi: I \rightarrow \mathbb{R}$ which satisfies

$$
\begin{aligned}
& \theta^{\prime}(s)=-p(s) \sin \varphi(s)-q(s) \cos \varphi(s) \\
& \varphi^{\prime}(s)=\tan \theta(s)(p(s) \cos \varphi(s)-q(s) \sin \varphi(s))+r \\
& \psi^{\prime}(s)=\sec \theta(s)(p(s) \cos \varphi(s)-q(s) \sin \varphi(s))
\end{aligned}
$$

we call the frame $\left\{\bar{\mu}_{1}, \bar{\mu}_{2}, \bar{\mu}_{3}, v\right\}$ a Bishop-type frame along framed base curve $\mathbb{R}^{4}$. Also, the formula is given by

$$
\left(\begin{array}{c}
v^{\prime}(s) \\
\bar{\mu}_{1}^{\prime}(s) \\
\bar{\mu}_{2}^{\prime}(s) \\
\bar{\mu}_{3}^{\prime}(s)
\end{array}\right)=\left(\begin{array}{cccc}
0 & -L(s) & -M(s) & -N(s) \\
L(s) & 0 & 0 & 0 \\
M(s) & 0 & 0 & 0 \\
N(s) & 0 & 0 & 0
\end{array}\right)\left(\begin{array}{l}
v(s) \\
\bar{\mu}_{1}(s) \\
\bar{\mu}_{2}(s) \\
\bar{\mu}_{3}(s)
\end{array}\right)
$$

where

$$
\begin{aligned}
& L(s)=l(s) \cos \theta(s) \cos \psi(s)-m(s) \cos \varphi(s) \sin \psi(s)+m(s) \sin \varphi(s) \cos \psi(s) \sin \theta(s) \\
& +n(s) \sin \varphi(s) \sin \psi(s)+n(s) \cos \varphi(s) \cos \psi(s) \sin \theta(s) \\
& M(s)=l(s) \cos \theta(s) \sin \psi(s)+m(s) \cos \varphi(s) \cos \psi(s)+m(s) \sin \varphi(s) \sin \psi(s) \sin \theta(s) \\
& -n(s) \sin \varphi(s) \cos \psi(s)+n(s) \cos \varphi(s) \sin \psi(s) \sin \theta(s) \\
& N(s)=-l(s) \sin \theta(s)+m(s) \sin \varphi(s) \cos \theta(s)+n(s) \cos \varphi(s) \cos \theta(s)
\end{aligned}
$$

and with equation 3.3 .

Corollary 3.4. According to the equation 3.3, there is

$$
\varphi^{\prime}(s)=\psi^{\prime}(s) \sin \theta(s)+r(s)
$$

relation between the Euler angles. 


\section{Special framed curves in $\mathbb{R}^{4}$ with Bishop frame}

\subsection{Framed rectifying curves}

Definition 4.1. Let $\left(\gamma, \bar{\mu}_{1}, \bar{\mu}_{2}, \bar{\mu}_{3}\right): I \rightarrow \mathbb{R}^{4} \times \Delta_{3}$ be a framed curve. $\gamma$ is called framed rectifying curve in $\mathbb{R}^{4}$ if its position vector $\gamma$ satisfies:

$$
\gamma(s)=\lambda_{1}(s) v(s)+\lambda_{2}(s) \bar{\mu}_{2}(s)+\lambda_{3}(s) \bar{\mu}_{3}(s)
$$

where $\lambda_{1}(s), \lambda_{2}(s)$ and $\lambda_{3}(s)$ are differentiable functions.

Theorem 4.2. Let $\left(\gamma, \bar{\mu}_{1}, \bar{\mu}_{2}, \bar{\mu}_{3}\right): I \rightarrow \mathbb{R}^{4} \times \Delta_{3}$ be a framed curve with non-zero framed curvatures. Then, $\gamma$ is a framed rectifying curve if and only if $\lambda_{2} M(s)+\lambda_{3} N(s)-\alpha(s)=0$, where $\lambda_{2}$ and $\lambda_{3}$ are real constants.

Proof. Assume $\gamma$ is a framed rectifying curve. By differentiating equation 4.1, we get

$$
\begin{aligned}
\alpha(s) v(s)= & \left(\lambda_{1}^{\prime}(s)+\lambda_{2}(s) M(s)+\lambda_{3}(s) N(s)\right) v(s)+\left(-L(s) \lambda_{1}(s)\right) \bar{\mu}_{1}(s) \\
& +\left(-M(s) \lambda_{1}(s)+\lambda_{2}^{\prime}(s)\right) \bar{\mu}_{2}(s)+\left(-N(s) \lambda_{1}(s)+\lambda_{3}^{\prime}(s)\right) \bar{\mu}_{3}(s)
\end{aligned}
$$

Then according to equation 4.2 , we have

$$
\begin{array}{lll}
L(s) \lambda_{1}(s) & = & 0 \\
M(s) \lambda_{1}(s)-\lambda_{2}^{\prime}(s) & = & 0 \\
N(s) \lambda_{1}(s)-\lambda_{3}^{\prime}(s) & = & 0 \\
\lambda_{1}^{\prime}(s)+\lambda_{2}(s) M(s)+\lambda_{3}(s) N(s) & = & \alpha(s)
\end{array}
$$

Since framed curvatures are non-zero, we get

$$
\left.\lambda_{1}(s)=0, \quad \lambda_{2}(s)=\lambda_{2}(\text { const } .), \lambda_{3}(s)=\lambda_{3} \text { (const. }\right)
$$

Therefore, by using last equation of 4.3 and equation 4.4 , we have

$$
\lambda_{2} M(s)+\lambda_{3} N(s)-\alpha(s)=0
$$

where $\lambda_{2}$ and $\lambda_{3}$ are real constants. Conversely, assume that the curvatures $(L, M, N, \alpha)$ satisfies the equation 4.5 . By considering, the vector $X \in \mathbb{R}^{4}$ given by

$$
X(s)=\gamma(s)-\lambda_{2} \bar{\mu}_{2}(s)-\lambda_{3} \bar{\mu}_{3}(s)
$$

By differentiating equation 4.6, we have

$$
X^{\prime}(s)=\alpha(s) v(s)-\left(\lambda_{2} M(s)+\lambda_{3} N(s)\right) v(s)
$$

By using equation 4.5 , we get

$$
X^{\prime}(s)=0
$$

Based on the equation 4.7, we conclude that $\gamma$ is congruent to a framed rectifying curve in $\mathbb{R}^{4}$.

Corollary 4.3. If $\gamma$ is a framed rectifying curve with non-zero curvatures in $\mathbb{R}^{4}$, then the curvatures of $\gamma$ have the following relationships

$$
\frac{\left(\frac{\alpha}{N}\right)^{\prime}}{\left(\frac{M}{N}\right)^{\prime}}=\text { constant } \text { or } \frac{\left(\frac{\alpha}{M}\right)^{\prime}}{\left(\frac{N}{M}\right)^{\prime}}=\text { constant }
$$

Proof. By using equation 4.5 and the derivative of equation 4.5 , we get 4.8 .

\subsection{Framed first osculating curves}

Definition 4.4. Let $\left(\gamma, \bar{\mu}_{1}, \bar{\mu}_{2}, \bar{\mu}_{3}\right): I \rightarrow \mathbb{R}^{4} \times \Delta_{3}$ be a framed curve. $\gamma$ is called framed first osculating curve in $\mathbb{R}^{4}$ if its position vector $\gamma$ satisfies:

$$
\gamma(s)=\varepsilon_{1}(s) v(s)+\varepsilon_{2}(s) \bar{\mu}_{1}(s)+\varepsilon_{3}(s) \bar{\mu}_{3}(s)
$$

for some functions $\varepsilon_{1}(s), \varepsilon_{2}(s), \varepsilon_{3}(s)$.

Theorem 4.5. Let $\left(\gamma, \bar{\mu}_{1}, \bar{\mu}_{2}, \bar{\mu}_{3}\right): I \rightarrow \mathbb{R}^{4} \times \Delta_{3}$ be a framed curve with non-zero framed curvatures. Then, $\gamma$ is a framed first osculating curve if and only if $\varepsilon_{2} L(s)+\varepsilon_{3} N(s)-\alpha(s)=0$ where $\varepsilon_{2}$ and $\varepsilon_{3}$ are real constants. 
Proof. Suppose that $\gamma$ is a framed first osculating curve. By differentiating equation 4.9, we get

$$
\begin{aligned}
\alpha(s) v(s)= & \left(\varepsilon_{1}^{\prime}(s)+\varepsilon_{2}(s) L(s)+\varepsilon_{3}(s) N(s)\right) v(s)+\left(-L(s) \varepsilon_{1}(s)+\varepsilon_{2}^{\prime}(s)\right) \bar{\mu}_{1}(s) \\
& -\varepsilon_{1}(s) M(s) \bar{\mu}_{2}(s)+\left(-N(s) \varepsilon_{1}(s)+\varepsilon_{3}^{\prime}(s)\right) \bar{\mu}_{3}(s)
\end{aligned}
$$

Therefore, we have

$$
\begin{array}{ll}
L(s) \varepsilon_{1}(s)-\varepsilon_{2}^{\prime}(s) & =0 \\
M(s) \varepsilon_{1}(s) & =0 \\
N(s) \varepsilon_{1}(s)+\varepsilon_{3}^{\prime}(s) & =0 \\
\varepsilon_{1}^{\prime}(s)+\varepsilon_{2}(s) L(s)+\varepsilon_{3}(s) N(s) & =\alpha(s)
\end{array}
$$

Since framed curvatures are non-zero, we get

$$
\varepsilon_{1}(s)=0, \quad \varepsilon_{2}=\varepsilon_{2}(\text { const. }), \varepsilon_{3}=\varepsilon_{3}(\text { const. })
$$

Consequently, by using last equation of 4.10 and equation 4.11 , we have

$$
\varepsilon_{2} L(s)+\varepsilon_{3} N(s)-\alpha(s)=0
$$

where $\varepsilon_{2}$ and $\varepsilon_{3}$ are real constants. Conversely, assume that the curvatures $(L, M, N, \alpha)$ satisfies the equation 4.12 . By considering, the vector $X \in \mathbb{R}^{4}$ given by

$$
X(s)=\gamma(s)-\varepsilon_{2} \bar{\mu}_{1}(s)-\varepsilon_{3} \bar{\mu}_{3}(s)
$$

By differentiating equation 4.13, we have

$$
X^{\prime}(s)=\alpha(s) v(s)-\left(\varepsilon_{2} L(s)+\varepsilon_{3} N(s)\right) v(s)
$$

By using equation 4.14 , we get

$$
X^{\prime}(s)=0
$$

By according to equation 4.15 , we conclude that $\gamma$ is congruent to a framed first osculating curve in $\mathbb{R}^{4}$.

Corollary 4.6. If $\gamma$ is a framed first osculating curve with non-zero curvatures in $\mathbb{R}^{4}$, then the curvatures of $\gamma$ have the following relationships

$$
\frac{\left(\frac{\alpha}{N}\right)^{\prime}}{\left(\frac{L}{N}\right)^{\prime}}=\text { constant } \quad \text { or } \frac{\left(\frac{\alpha}{L}\right)^{\prime}}{\left(\frac{N}{L}\right)^{\prime}}=\text { constant }
$$

Proof. By using equation 4.12 and the derivative of equation 4.12, we get 4.16.

\subsection{Framed second osculating curves}

Definition 4.7. Let $\left(\gamma, \bar{\mu}_{1}, \bar{\mu}_{2}, \bar{\mu}_{3}\right): I \rightarrow \mathbb{R}^{4} \times \Delta_{3}$ be a framed curve. $\gamma$ is called framed second osculating curve in $\mathbb{R}^{4}$ if its position vector $\gamma$ satisfies:

$$
\gamma(s)=\eta_{1}(s) v(s)+\eta_{2}(s) \bar{\mu}_{1}(s)+\eta_{3}(s) \bar{\mu}_{2}(s)
$$

for some functions $\eta_{1}(s), \eta_{2}(s), \eta_{3}(s)$.

Theorem 4.8. Let $\left(\gamma, \bar{\mu}_{1}, \bar{\mu}_{2}, \bar{\mu}_{3}\right): I \rightarrow \mathbb{R}^{4} \times \Delta_{3}$ be a framed curve with non-zero framed curvatures. Then, $\gamma$ is a framed second osculating curve if and only if $\eta_{2} L(s)+\eta_{3} M(s)-\alpha(s)=0$ where $\eta_{2}$ and $\eta_{3}$ are real constants.

Proof. Its proof is done in a similar way to Theorem 4.5 .

Corollary 4.9. If $\gamma$ is a framed second osculating curve with non-zero curvatures in $\mathbb{R}^{4}$, then the curvatures of $\gamma$ have the following relationships

$$
\frac{\left(\frac{\alpha}{M}\right)^{\prime}}{\left(\frac{L}{M}\right)^{\prime}}=\text { constant } \quad \text { or } \quad \frac{\left(\frac{\alpha}{L}\right)^{\prime}}{\left(\frac{M}{L}\right)^{\prime}}=\text { constant }
$$




\subsection{Framed normal curves}

Definition 4.10. Let $\left(\gamma, \bar{\mu}_{1}, \bar{\mu}_{2}, \bar{\mu}_{3}\right): I \rightarrow \mathbb{R}^{4} \times \Delta_{3}$ be a framed curve. $\gamma$ is called framed normal curve in $\mathbb{R}^{4}$ if its position vector $\gamma$ satisfies:

$$
\gamma(s)=\delta_{1}(s) \bar{\mu}_{1}(s)+\delta_{2}(s) \bar{\mu}_{2}(s)+\delta_{3}(s) \bar{\mu}_{3}(s)
$$

for some functions $\delta_{1}(s), \delta_{2}(s), \delta_{3}(s)$.

Theorem 4.11. Let $\left(\gamma, \bar{\mu}_{1}, \bar{\mu}_{2}, \bar{\mu}_{3}\right): I \rightarrow \mathbb{R}^{4} \times \Delta_{3}$ be a framed curve with non-zero framed curvatures. Then, $\gamma$ is a framed normal curve if and only if $\left.\delta_{1} L(s)\right)+\delta_{2} M(s)+\delta_{3} N(s)-\alpha(s)=0$ where $\delta_{1}, \delta_{2}, \delta_{3}$ are real constants.

Proof. Suppose that $\gamma$ is a framed normal curve. By differentiating equation 4.17, we get

$$
\begin{aligned}
\alpha(s) v(s)= & \left(\delta_{1}(s) L(s)+\delta_{2}(s) M(s)+\delta_{3}(s) N(s)\right) v(s)+\delta_{1}^{\prime}(s) \bar{\mu}_{1}(s) \\
& +\delta_{2}^{\prime} \bar{\mu}_{2}(s)+\delta_{3}^{\prime}(s) \bar{\mu}_{3}(s)
\end{aligned}
$$

Therefore, we have

$$
\begin{aligned}
& \delta_{1}^{\prime}(s)=0 \\
& \delta_{2}^{\prime}(s)=0 \\
& \delta_{3}^{\prime}(s)=0 \\
& \delta_{1}(s) L(s)+\delta_{2}(s) M(s)+\delta_{3}(s) N(s)=\alpha(s)
\end{aligned}
$$

Then, we have

$$
\delta_{1}(s)=\delta_{1} \text { (const.), } \delta_{2}=\delta_{2}(\text { const. }), \delta_{3}=\delta_{3} \text { (const.) }
$$

Consequently, by using last equation of 4.18 and equation 4.19 , we have

$$
\delta_{1} L(s)+\delta_{2} M(s)+\delta_{3} N(s)-\alpha(s)=0
$$

where $\delta_{1}, \delta_{2}$ and $\delta_{3}$ are real constants. Conversely, assume that the curvatures $(L, M, N, \alpha)$ satisfies the equation 4.20. By considering, the vector $X \in \mathbb{R}^{4}$ given by

$$
X(s)=\gamma(s)-\delta_{1} \bar{\mu}_{1}(s)-\delta_{2} \bar{\mu}_{2}(s)-\delta_{3} \bar{\mu}_{3}(s)
$$

By differentiating equation 4.21 , we have

$$
X^{\prime}(s)=\alpha(s) v(s)-\left(\delta_{1} L(s)+\delta_{2} M(s)+\delta_{3} N(s)\right) v(s)
$$

Then, we get

$$
X^{\prime}(s)=0
$$

By according to equation 4.22 , we conclude that $\gamma$ is congruent to a framed normal curve in $\mathbb{R}^{4}$.

\section{Conclusion}

In this study, we defined framed curves in four-dimensional Euclidean space. In addition, we gave Bishop-type frame of framed curves in four-dimensional Euclidean space. Actually, the Frenet-type frame of framed curves can give in four-dimensional Euclidean space. In addition, we investigate framed rectifying, normal and osculating curves. Thus, since the four-dimensional frame of framed curve, all framed curve studies can be extended to this space.

\section{Acknowledgements}

The authors would like to express their sincere thanks to the editor and the anonymous reviewers for their helpful comments and suggestions.

\section{Funding}

There is no funding for this work.

\section{Availability of data and materials}

Not applicable.

\section{Competing interests}

The authors declare that they have no competing interests.

\section{Author's contributions}

All authors contributed equally to the writing of this paper. All authors read and approved the final manuscript. 


\section{References}

[1] S. Honda, M. Takahashi, Framed curves in the Euclidean space, Adv. Geo., 16(3) (2016), 265-276, https://doi.org/10.1515/advgeom-2015-0035.

[2] T. Fukunaga, M. Takahashi, Existence conditions of framed curves for smooth curves, J. Geo., 108 (2017), 763-774, https://doi.org/10.1007/s00022-017-

[3] S. Honda, M. Takahashi, Evolutes and focal surfaces of framed immersions in the Euclidean space, Proceedings of the Royal Society of Edinburgh: Section A Mathematics, 150(1) (2020), 497-516, https://doi.org/10.1017/prm.2018.84.

[4] Y. Wang Y, D. Pei, R. Gao, Generic properties of framed rectifying curves, Mathematics, 7(1)(2019), 37.

[5] B. D. Yazıcı, S. Ö. Karakuş, M. Tosun, On the classification of framed rectifying curves in Euclidean space, Math. Meth. Appl. Sci., (2021), 1-10, https://doi.org/10.1002/mma.7561.

[6] B. D. Yazıcı, S. Ö. Karakus, M. Tosun, Framed normal curves in Euclidean space, Tbilisi Math. J., (2021), 27-37, https://doi.org/10.2478/9788395793882003.

[7] S. Honda, M. Takahashi, Bertrand and Mannheim curves of framed curves in the 3-dimensional Euclidean space, Turkish Journal of Mathematics, 44 (2020), 883-899, https://doi.org/10.3906/mat-1905-63.

[8] L. R. Bishop, There is more than one way to frame a curve, Amer. Math. Monthly, 82(3) (1975), 246-251, https://doi.org/10.2307/2319846.

[9] F. Gökçelik, Z. Bozkurt, İ. Gök, F. N. Ekmekçi, Y. Yayli, Parallel transport frame in 4-dimensional Euclidean space, Caspian J. of Math. Sci., 3 (2014), 91-103.

[10] F. Ateş, I. Gök, F.N. Ekmekçi, Y. Yaylı, Characterizations of inclined curves according to parallel transport frame in $E^{4}$ and Bishop Frame in $E^{3}$, Konuralp Journal of Mathematics, 7 (2019), 16-24.

[11] T. Körpinar, E. Turhan, Biharmonic curves according to parallel transport frame in E4, Bol. Soc. Paran. Mat., 31 (2)(2013), 213-217, https://doi.org/10.5269/bspm.v31i2.17669.

[12] Z. Özdemir, I. Gök, F. N. Ekmekçi, Y. Yayl1, A new approach on type-3 slant helix in $E^{4}$, Gen. Math. Notes, 28(1) (2015), 40-49.

[13] A. J. Hanson, H. Ma, Parallel Transport Approach to Curve Framing, Tech. Math. Rep. 425, Indiana University Computer science Department, 1995.

[14] M. Z. Williams, F.M. Stein, A triple product of vectors in four-space, Math Mag., 37 (1964), 230-235. 